\title{
Pizzicato excitation for wearable energy harvesters
}

\section{Michele Pozzi and Meiling Zhu}

A new technique based on the plucking of flexible piezoelectric material can be used to boost energy harvested to power portable electronic devices.

Recent decades have witnessed the miniaturization of electronic devices. The reduction in the feature size of gadgets' components is beneficial not only because we can pack many transistors in a small space, but also because compact devices consume less power. Currently, a good proportion of the volume and weight of portable gadgets is taken up by batteries, which have a size dictated by a difficult compromise between portability and battery life. However, our dependence on batteries may be close to an end. Since miniaturized electronics do not require much power, it is becoming viable to generate the necessary energy within the device itself or with portable energy harvesters (EHs). These systems supply an inexhaustible amount of energy by converting vibrations or other mechanical movement, thermal gradients, or electromagnetic radiation into electricity.

Among the first EHs to be studied were scavengers of the vibrational energy naturally present in many environments. These devices power wireless sensor nodes that constantly monitor important environmental parameters for an extended period of time without maintenance or battery replacement. Most of these vibrational-energy harvesters are based on piezoelectric transducers, devices capable of converting energy between physical domains such as electrical into mechanical or vice versa.

When it comes to wearable EHs, that is, devices that can harvest the energy generated naturally by the human body (through walking, for example) we are faced with a mismatch. Human movements have characteristically low frequencies (up to a few hertz) while piezoelectric technology can produce sizable power at high frequencies only. A viable solution is to employ frequency up-conversion techniques. These methods are used to convert a low input frequency into a high output one through mechanisms such as gears and cams or through impact or plucking. In pianos, up-conversion is achieved when hammers strike the wires that are then free to vibrate at resonance. For

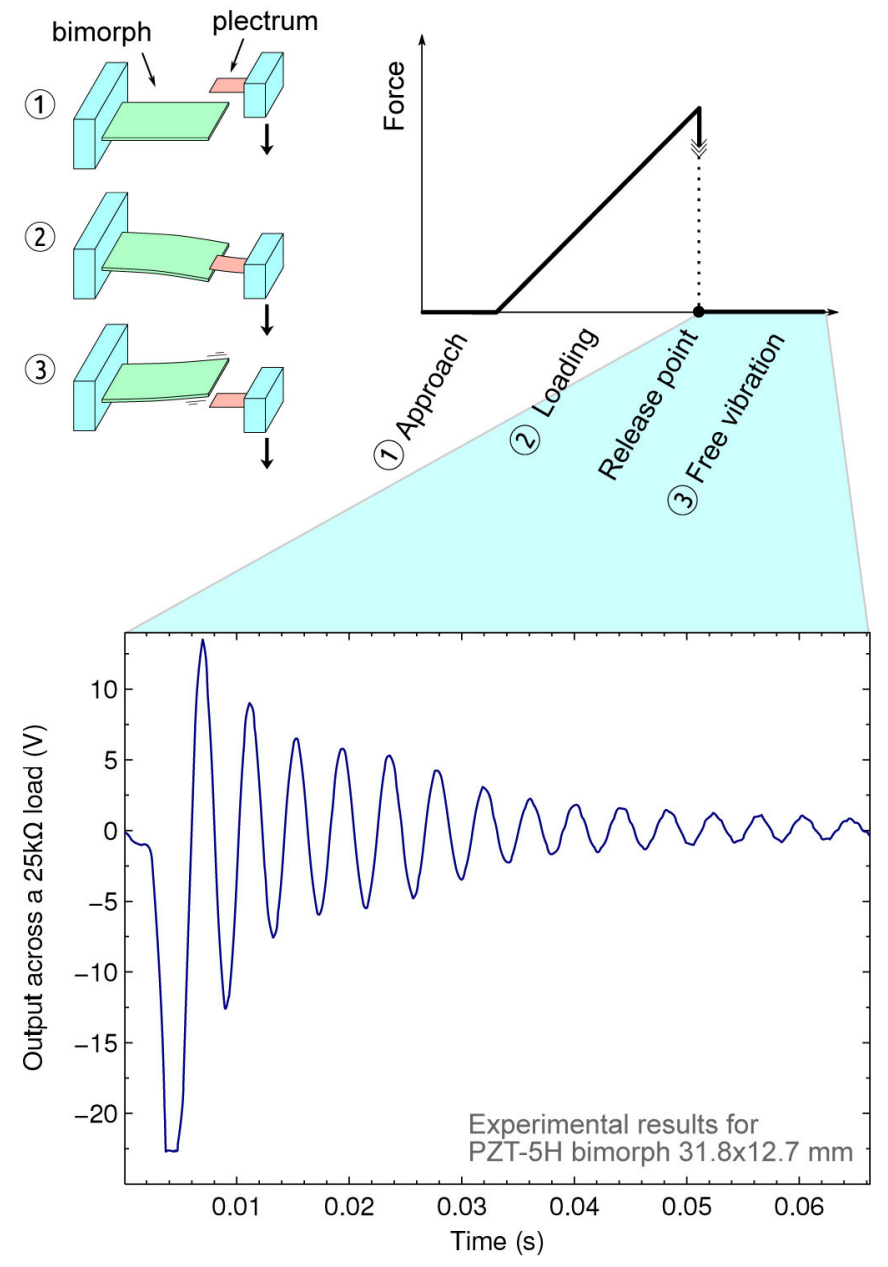

Figure 1. The pizzicato up-frequency conversion. A plectrum approaches a bimorph (1) and mechanical energy is stored elastically after contact (2). Upon release, the bimorph vibrates at resonance (3) and voltage is produced across an electrical load (lower plot). PZT5H: A 'flavor' of PZT (lead zirconate titanate) characterized by a specific chemical composition. 


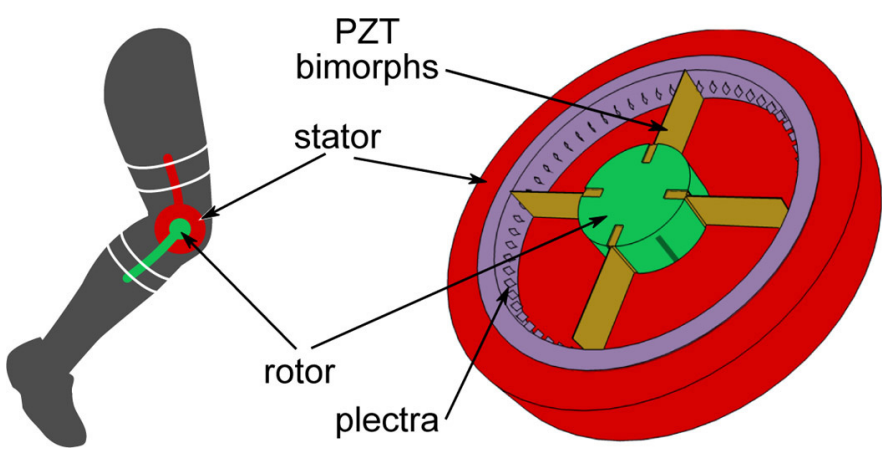

Figure 2. The knee-joint harvester. The plectra-carrying outer casing is fixed to the thigh of the wearer, and the bimorphs-bearing central hub is fixed to the shank. During normal walking, bimorphs are plucked by plectra and convert mechanical energy into electrical energy.

piezoelectric EHs, this approach was pioneered by Umeda and others with a steel ball impacting a piezoelectric disc. ${ }^{1}$ In harpsichords and guitars, plectra slowly deflect the chords that vibrate at resonance upon release. This plucking of the strings of a musical instrument is known as the pizzicato technique, a concept that can also be applied to up-conversion in piezoelectric transducers.

We are currently interested in the study of wearable EHs that can be used to power personal electronic gadgets (mobile phones, blood-sugar monitors, and others). To boost the energy harvested, we employ the pizzicato up-conversion technique using plectra and piezoelectric bimorphs. (The latter are devices with two separate layers of piezo-material that bend when energized and, conversely, generate electrical energy when deformed.) The upper panel of Figure 1 shows an implementation of the plucking excitation. A plectrum and a bimorph move relatively to each other. After contact, both deflect and mechanical energy is stored as elastic deformation. Electrical energy is produced by the piezoelectric effect both during deflection and, in greater quantity, in the resonance vibrations that follow release. From the experimental curve of the voltage generated as a function of time, the energy produced can be calculated $(38 \mu \mathrm{J}$ in the event reproduced in the figure).

With this principle as a starting point, we numerically modeled the response of a bimorph and the power generated by plucking. We focused on the effects of several parameters, such as electrical loading, the interval between subsequent plucking actions, and the speed of deflection. ${ }^{2}$ With this knowledge, we designed and prototyped a wearable knee-joint harvester. ${ }^{3}$ The device is made of two parts: an internal hub fixed to the shank of the wearer and an outer casing fixed to the thigh (see Figure 2). As the person walks, the hub and casing rotate relatively to each other so that a large number of plectra (on the casing) pass in front of each bimorph (on the hub) and pluck it. Every time this happens, each bimorph starts vibrating and produces energy, until all of the mechanical energy is either converted or dissipated. Thus, energy follows a staircase-like curve as a function of time, with each step upwards associated to a plucking event. The height of the riser measures the energy produced, while the length of the tread is linked to the spacing between plectra.

In the case of a multi-bimorph harvester, an electrical complication arises. Since the time of release of each bimorph has a random component, the voltage waveforms output by the devices (similar to those in the lower panel of Figure 1) will not be in phase. Since the signal oscillates between positive and negative values, if the electrodes of the bimorphs were connected together, we would see electrical charges transferred among the devices with a significant penalty in power output. Therefore, it is necessary to rectify each bimorph's output individually before bringing them together. Fortunately, small rectifying bridges are available as integrated circuits, and the output of a large number of bimorphs can be conditioned with very compact electronics.

The advantages of our design include mechanical simplicity. In fact, precision engineering is not required to produce the harvester because standard manufacturing tolerances are adequate. While the current prototype can accommodate a maximum of four bimorphs, an industrially manufactured device would not have this limitation. We estimate that tens of $\mathrm{mW}$ of useful power can be generated before further design optimization is required.

The drastic reduction in size and power requirements of portable electronics has spurred intense research in energy harvesting. We proposed a knee-joint harvester based on a pizzicato frequency up-conversion technique for wearable power generation. In addition, we designed a prototype capable of output powers in the order of $\mathrm{mW}$, and we anticipate that larger powers can be made available with design improvements and further development. In the future, we plan to conduct tests using bio-mechanical gait data and trials on human subjects. We will also investigate a re-design of the harvester with magnetic plectra.

\section{Author Information}

Michele Pozzi and Meiling Zhu

Cranfield University

Cranfield, United Kingdom 
Michele Pozzi holds a diploma in physics, a MSc in materials science and technology, and a PhD in mechatronics. Previously, he was an assistant professor at Sirindhorn International Institute of Technology, Thammasat University in Thailand and a researcher at Newcastle University in the UK. He is currently a research fellow in energy harvesting.

Meiling Zhu received her PhD from Southeast University, Nanjing, China in 1995. She is currently working with the Department of Materials at the School of Applied Science of Cranfield University as a senior research fellow. Her scientific interests lie in the field of piezoelectric sensing and actuation, and energy harvesting. Her expertise is mainly in design, analysis, modeling and simulation, prototyping, and applications of macro/micro sized piezoelectric devices.

\section{References}

1. M. Umeda, K. Nakamura, and S. Ueha, Analysis of the transformation of mechanical impact energy to electric energy using piezoelectric vibrator, Jpn. J. Appl. Phys. 35, pp. 3267-3273, 1996. doi:10.1143/JJAP.35.3267

2. M. Pozzi and M. Zhu, Plucked piezoelectric bimorphs for knee-joint energy harvesting: modeling and experimental validation, Smart Mater. Struct. 20 (5), p. 055007, 2011. doi:10.1088/0964-1726/20/5/055007

3. M. Pozzi and M. Zhu, Plucked piezoelectric bimorphs for energy harvesting applications, Proc. SPIE 8066, 2011. Accepted as paper 8066-42 at the SPIE Microtechnol.: Smart Sensors, Actuators and MEMS Conf. in Prague, Czech Republic, 18-20 April 2011. 